\title{
Fuzzy Ostrowski type inequalities
}

\author{
GEORGE A. ANASTASSIOU \\ Department of Mathematical Sciences \\ University of Memphis, Memphis, TN 38152 U.S.A. \\ E-mail: ganastss@memphis.edu
}

\begin{abstract}
We present optimal upper bounds for the deviation of a fuzzy continuous function from its fuzzy average over $[a, b] \subset \mathbb{R}$, error is measured in the $D$-fuzzy metric. The established fuzzy Ostrowski type inequalities are sharp, in fact attained by simple fuzzy real number valued functions. These inequalities are given for fuzzy Hölder and fuzzy differentiable functions and these facts are reflected in their right-hand sides.
\end{abstract}

Mathematical subject classification: 26D07, 26D15, 26E50.

Key words: Fuzzy inequalities, Ostrowski inequality, Fuzzy real analysis.

\section{Introduction}

Ostrowski inequality (see [8]) has as follows

$$
\left|\frac{1}{b-a} \int_{a}^{b} f(y) d y-f(x)\right| \leq\left(\frac{1}{4}+\frac{\left(x-\frac{a+b}{2}\right)^{2}}{(b-a)^{2}}\right)(b-a)\left\|f^{\prime}\right\|_{\infty},
$$

where $f \in C^{1}([a, b]), x \in[a, b]$. Inequality $(*)$ is sharp, see [1].

Since 1938 when A. Ostrowski proved his famous inequality, see [8], many people have been working about and around it, in many different directions and with a lot of applications in Numerical Analysis and Probability, etc.

One of the most notable works extending Ostrowski's inequality is the work of A.M. Fink, see [6]. The author in [1] continued that tradition.

This current article is mainly motivated by [1], [6], [8], [11] and extends Ostrowski type inequalities into the fuzzy setting, as fuzzyness is a natural reality 
genuine feature different than randomness and determinism. To the best of our knowledge this is the first attempt of such extension into the fuzzy environment, hoping to find wide continuations and lots of applications.

\section{Background}

We start with

Definition 1 (see [10]). Let $\mu: \mathbb{R} \rightarrow[0,1]$ with the following properties:

(i) is normal, i.e., $\exists x_{0} \in \mathbb{R} ; \mu\left(x_{0}\right)=1$.

(ii) $\mu(\lambda x+(1-\lambda) y) \geq \min \{\mu(x), \mu(y)\}, \forall x, y \in \mathbb{R}, \forall \lambda \in[0,1]$ ( $\mu$ is called a convex fuzzy subset).

(iii) $\mu$ is upper semicontinuous on $\mathbb{R}$, i.e., $\forall x_{0} \in \mathbb{R}$ and $\forall \varepsilon>0, \exists$ neighborhood $V\left(x_{0}\right): \mu(x) \leq \mu\left(x_{0}\right)+\varepsilon, \forall x \in V\left(x_{0}\right)$.

(iv) The set $\overline{\operatorname{supp}(\mu)}$ is compact in $\mathbb{R}$ (where $\operatorname{supp}(\mu):=\{x \in \mathbb{R} ; \mu(x)>0\}$ ).

We call $\mu$ a fuzzy real number. Denote the set of all $\mu$ with $\mathbb{R}_{\mathcal{F}}$.

E.g., $\chi_{\left\{x_{0}\right\}} \in \mathbb{R}_{\mathcal{F}}$, for any $x_{0} \in \mathbb{R}$, where $\mathcal{X}_{\left\{x_{0}\right\}}$ is the characteristic function at $x_{0}$.

For $0<r \leq 1$ and $\mu \in \mathbb{R}_{\mathcal{F}}$ define $[\mu]^{r}:=\{x \in \mathbb{R}: \mu(x) \geq r\}$ and

$$
[\mu]^{0}:=\overline{\{x \in \mathbb{R}: \mu(x)>0\}} .
$$

Then it is well known that for each $r \in[0,1],[\mu]^{r}$ is a closed and bounded interval of $\mathbb{R}$. For $u, v \in \mathbb{R}_{\mathcal{F}}$ and $\lambda \in \mathbb{R}$, we define uniquely the sum $u \oplus v$ and the product $\lambda \odot u$ by

$$
[u \oplus v]^{r}=[u]^{r}+[v]^{r}, \quad[\lambda \odot u]^{r}=\lambda[u]^{r}, \quad \forall r \in[0,1],
$$

where $[u]^{r}+[v]^{r}$ means the usual addition of two intervals (as subsets of $\mathbb{R}$ ) and $\lambda[u]^{r}$ means the usual product between a scalar and a subset of $\mathbb{R}$ (see, e.g., [10]). Notice $1 \odot u=u$ and it holds $u \oplus v=v \oplus u, \lambda \odot u=u \odot \lambda$. If $0 \leq r_{1} \leq r_{2} \leq 1$ then $[u]^{r_{2}} \subseteq[u]^{r_{1}}$. Actually $[u]^{r}=\left[u_{-}^{(r)}, u_{+}^{(r)}\right]$, where $u_{-}^{(r)} \leq u_{+}^{(r)}, u_{-}^{(r)}, u_{+}^{(r)} \in \mathbb{R}$, $\forall r \in[0,1]$. 
Define

$$
D: \mathbb{R}_{\mathcal{F}} \times \mathbb{R}_{\mathcal{F}} \rightarrow \mathbb{R}_{+} \cup\{0\}
$$

by

$$
D(u, v):=\sup _{r \in[0,1]} \max \left\{\left|u_{-}^{(r)}-v_{-}^{(r)}\right|,\left|u_{+}^{(r)}-v_{+}^{(r)}\right|\right\},
$$

where $[v]^{r}=\left[v_{-}^{(r)}, v_{+}^{(r)}\right] ; u, v \in \mathbb{R}_{\mathcal{F}}$. We have that $D$ is a metric on $\mathbb{R}_{\mathcal{F}}$. Then $\left(\mathbb{R}_{\mathcal{F}}, D\right)$ is a complete metric space, see [10], with the properties

$$
\begin{aligned}
& D(u \oplus w, v \oplus w)=D(u, v), \quad \forall u, v, w \in \mathbb{R}_{\mathcal{F}}, \\
& D(k \odot u, k \odot v)=|k| D(u, v), \quad \forall u, v \in \mathbb{R}_{\mathcal{F}}, \forall k \in \mathbb{R}, \\
& D(u \oplus v, w \oplus e) \leq D(u, w)+D(v, e), \quad \forall u, v, w, e \in \mathbb{R}_{\mathcal{F}} .
\end{aligned}
$$

We need

Lemma 1 (Lemma 2.2 of [5]). For any $a, b \in \mathbb{R}: a, b \geq 0$ and any $u \in \mathbb{R}_{\mathcal{F}}$ we have

$$
D(a \odot u, b \odot u) \leq|a-b| \cdot D(u, \tilde{o}),
$$

where $\tilde{o} \in \mathbb{R}_{\mathcal{F}}$ is defined by $\tilde{o}:=\mathcal{X}_{\{0\}}$.

We also need

Definition 2 (see [10]). Let $x, y \in \mathbb{R}_{\mathcal{F}}$. If there exists a $z \in \mathbb{R}_{\mathcal{F}}$ such that $x=y+z$, then we call $z$ the $H$-difference of $x$ and $y$, denoted by $z:=x-y$.

Definition 3 (Definition 3.3 of [10]). Let $T:=\left[x_{0}, x_{0}+\beta\right] \subset \mathbb{R}$, with $\beta>0$. A function $f: T \rightarrow \mathbb{R}_{\mathcal{F}}$ is $H$-differentiable at $x \in T$ if there exists a $f^{\prime}(x) \in \mathbb{R}_{\mathcal{F}}$ such that the limits (with respect to metric $D$ )

$$
\lim _{h \rightarrow 0^{+}} \frac{f(x+h)-f(x)}{h}, \quad \lim _{h \rightarrow 0^{+}} \frac{f(x)-f(x-h)}{h}
$$

exist and are equal to $f^{\prime}(x)$. We call $f^{\prime}$ the derivative or $H$-derivative of $f$ at $x$. If $f$ is $H$-differentiable at any $x \in T$, we call $f$ differentiable or $H$-differentiable and it has $H$-derivative over $T$ the function $f^{\prime}$.

The last definition was given first by M. Puri and D. Ralescu [9]. 
We use a particular case of the Fuzzy Henstock integral $\left(\delta(x)=\frac{\delta}{2}\right)$ introduced in [10], Definition 2.1.

That is,

Definition 4 (Definition 13.14 of [7], p. 644). Let $f:[a, b] \rightarrow \mathbb{R}_{\mathcal{F}}$. We say that $f$ is Fuzzy-Riemann integrable to $I \in \mathbb{R}_{\mathcal{F}}$ if for any $\varepsilon>0$, there exists $\delta>0$ such that for any division $P=\{[u, v] ; \xi\}$ of $[a, b]$ with the norms $\Delta(P)<\delta$, we have

$$
D\left(\sum_{P} *(v-u) \odot f(\xi), I\right)<\varepsilon,
$$

where $\sum^{*}$ denotes the fuzzy summation. We choose to write

$$
I:=(F R) \int_{a}^{b} f(x) d x .
$$

We also call an $f$ as above (F R)-integrable.

Corollary 1 (Corollary 13.2 of [7]). If $f \in C\left([a, b], \mathbb{R}_{\mathcal{F}}\right)$ then $f$ is $(F R)$ integrable on $[a, b]$.

We also need

Lemma 2 (Lemma 1 of [2]). If $f, g:[a, b] \subseteq \mathbb{R} \rightarrow \mathbb{R}_{\mathcal{F}}$ are fuzzy continuous (with respect to metric $D$ ), then the function $F:[a, b] \rightarrow \mathbb{R}_{+} \cup\{0\}$ defined by $F(x):=D(f(x), g(x))$ is continuous on $[a, b]$, and

$$
D\left((F R) \int_{a}^{b} f(u) d u,(F R) \int_{a}^{b} g(u) d u\right) \leq \int_{a}^{b} D(f(x), g(x)) d x .
$$

We mention

Lemma 3 (Lemma 3 of [2]). Let $f:[a, b] \subseteq \mathbb{R} \rightarrow \mathbb{R}_{\mathcal{F}}$ be fuzzy continuous. Then

$$
(F R) \int_{a}^{x} f(t) d t \text { is a fuzzy continuous function in } x \in[a, b] .
$$

We use the Fuzzy Taylor formula. 
Theorem 1 (Theorem 1 of [2]). Let $T:=\left[x_{0}, x_{0}+\beta\right] \subset \mathbb{R}$, with $\beta>0$. We assume that $f^{(i)}: T \rightarrow \mathbb{R}_{\mathcal{F}}$ are $H$-differentiable for all $i=0,1, \ldots, n-$ 1 , for any $x \in T$. (I.e., there exist in $\mathbb{R}_{\mathcal{F}}$ the H-differences $f^{(i)}(x+h)-$ $f^{(i)}(x), f^{(i)}(x)-f^{(i)}(x-h), i=0,1, \ldots, n-1$ for all small $h: 0<h<\beta$. Furthermore there exist $f^{(i+1)}(x) \in \mathbb{R}_{\mathcal{F}}$ such that the limits in D-distance exist and

$$
f^{(i+1)}(x)=\lim _{h \rightarrow 0^{+}} \frac{f^{(i)}(x+h)-f^{(i)}(x)}{h}=\lim _{h \rightarrow 0^{+}} \frac{f^{(i)}(x)-f^{(i)}(x-h)}{h},
$$

for all $i=0,1, \ldots, n-1$.) Also we assume that $f^{(n)}$, is fuzzy continuous on $T$. Then for $s \geq a, s, a \in T$ we obtain

$$
\begin{aligned}
f(s)= & f(a) \oplus f^{\prime}(a) \odot(s-a) \oplus f^{\prime \prime}(a) \odot \frac{(s-a)^{2}}{2 !} \\
& \oplus \cdots \oplus f^{(n-1)}(a) \odot \frac{(s-a)^{n-1}}{(n-1) !} \oplus R_{n}(a, s),
\end{aligned}
$$

where

$$
\left.R_{n}(a, s):=(F R) \int_{a}^{s}\left(\int_{a}^{s_{1}} \cdots\left(\int_{a}^{s_{n-1}} f^{(n)}\left(s_{n}\right) d s_{n}\right) d s_{n-1}\right) \cdots\right) d s_{1} .
$$

Here $R_{n}(a, s)$ is fuzzy continuous on $T$ as a function of $s$.

We use

Proposition 1 (Proposition 1 of [4]). Let $F(t):=t^{n} \odot u, t \geq 0, n \in \mathbb{N}$, and $u \in \mathbb{R}_{\mathcal{F}}$ be fixed. Then (the $H$-derivative)

$$
F^{\prime}(t)=n t^{n-1} \odot u .
$$

In particular when $n=1$ then $F^{\prime}(t)=u$.

We mention

Proposition 2 (Proposition 6 of [4]). Let I be an open interval of $\mathbb{R}$ and let $f: I \rightarrow \mathbb{R}_{\mathcal{F}}$ be $H$-fuzzy differentiable, $c \in \mathbb{R}$. Then

$$
(c \odot f)^{\prime} \text { exists and }(c \odot f)^{\prime}=c \odot f^{\prime}(x) .
$$

We use the "Fuzzy Mean Value Theorem". 
Theorem 2 (Theorem 1 of [4]). Let $f:[a, b] \rightarrow \mathbb{R}_{\mathcal{F}}$ be a fuzzy differentiable function on $[a, b]$ with $H$-fuzzy derivative $f^{\prime}$ which is assumed to be fuzzy continuous. Then

$$
D(f(d), f(c)) \leq(d-c) \sup _{t \in[c, d]} D\left(f^{\prime}(t), \tilde{o}\right),
$$

for any $c, d \in[a, b]$ with $d \geq c$.

We finally need the "Univariate Fuzzy Chain Rule".

Theorem 3 (Theorem 2 of [4]). Let I be a closed interval in $\mathbb{R}$. Here $g: I \rightarrow$ $\zeta:=g(I) \subseteq \mathbb{R}$ is differentiable, and $f: \zeta \rightarrow \mathbb{R}_{\mathcal{F}}$ is $H$-fuzzy differentiable. Assume that $g$ is strictly increasing. Then $(f \circ g)^{\prime}(x)$ exists and

$$
(f \circ g)^{\prime}(x)=f^{\prime}(g(x)) \odot g^{\prime}(x), \quad \forall x \in I .
$$

\section{Results}

We give the following

Theorem 4. Let $f \in C\left([a, b], \mathbb{R}_{\mathcal{F}}\right)$, the space of fuzzy continuous functions, $x \in[a, b]$ be fixed. We assume that $f$ fulfills the Hölder condition

$$
D(f(y), f(z)) \leq L_{f} \cdot|y-z|^{\alpha}, \quad 0<\alpha \leq 1, \forall y, z \in[a, b],
$$

for some $L_{f}>0$. Then

$$
\begin{gathered}
D\left(\frac{1}{b-a} \odot(F R) \int_{a}^{b} f(y) d y, f(x)\right) \\
\quad \leq L_{f}\left(\frac{(x-a)^{\alpha+1}+(b-x)^{\alpha+1}}{(\alpha+1)(b-a)}\right) .
\end{gathered}
$$

Proof. We have that

$$
\begin{aligned}
D & \left(\frac{1}{b-a} \odot(F R) \int_{a}^{b} f(y) d y, f(x)\right) \\
\quad & =D\left(\frac{1}{b-a} \odot(F R) \int_{a}^{b} f(y) d y, \frac{1}{b-a} \odot(F R) \int_{a}^{b} f(x) d y\right)
\end{aligned}
$$




$$
\begin{aligned}
& =\frac{1}{b-a} D\left(\int_{a}^{b} f(y) d y, \int_{a}^{b} f(x) d y\right) \\
& \underset{\leq}{\text { by Lemma 2) }} \frac{1}{b-a} \int_{a}^{b} D(f(y), f(x)) d y \\
& \leq \frac{L_{f}}{b-a} \int_{a}^{b}|y-x|^{\alpha} d y=\left(\frac{L_{f}}{b-a}\right)\left(\frac{(x-a)^{\alpha+1}+(b-x)^{\alpha+1}}{\alpha+1}\right) .
\end{aligned}
$$

Optimality of (1) comes next.

Proposition 3. Inequality (1) is sharp, in fact, attained by $f^{*}(y):=|y-x|^{\alpha} \odot u$, $0<\alpha \leq 1$, with $u \in \mathbb{R}_{\mathcal{F}}$ fixed. Here $x, y \in[a, b]$.

Proof. Clearly $f^{*} \in C\left([a, b], \mathbb{R}_{\mathcal{F}}\right)$ : for letting $y_{n} \rightarrow y, y_{n} \in[a, b]$, then

$$
D\left(f^{*}\left(y_{n}\right), f^{*}(y)\right)=D\left(\left|y_{n}-x\right|^{\alpha} \odot u,|y-x|^{\alpha} \odot u\right)
$$

(by Lemma 1)

$$
\leq \quad|| y_{n}-\left.x\right|^{\alpha}-|y-x|^{\alpha} \mid D(u, \tilde{o}) \rightarrow 0, \text { as } n \rightarrow+\infty \text {. }
$$

Furthermore

$$
\begin{gathered}
D\left(f^{*}(y), f^{*}(z)\right)=D\left(|y-x|^{\alpha} \odot u,|z-x|^{\alpha} \odot u\right) \\
\quad \underset{\leq}{(\text { by Lemma 1) }} \| y-\left.x\right|^{\alpha}-|z-x|^{\alpha} \mid D(u, \tilde{o}) \\
\leq|| y-x|-| z-\left.x\right|^{\alpha} D(u, \tilde{o}) \leq|y-z|^{\alpha} D(u, \tilde{o}) .
\end{gathered}
$$

That is, for $L_{f^{*}}:=D(u, \tilde{o})$ we get

$$
D\left(f^{*}(y), f^{*}(z)\right) \leq L_{f^{*}}|y-z|^{\alpha}, \quad 0<\alpha \leq 1 \text {, any } y, z \in[a, b] .
$$

So that $f^{*}$ is a Hölder function. 
Finally we have

$$
\begin{aligned}
D & \left(\frac{1}{b-a} \odot(F R) \int_{a}^{b} f^{*}(y) d y, f^{*}(x)\right) \\
& =D\left(\frac{1}{b-a} \odot(F R) \int_{a}^{b}\left(|y-x|^{\alpha} \odot u\right) d y, \tilde{o}\right) \\
& =\frac{1}{b-a} \cdot D\left((F R) \int_{a}^{b}\left(|y-x|^{\alpha} \odot u\right) d y, \tilde{o}\right) \\
& =\frac{1}{b-a} D\left(\left(\int_{a}^{b}|y-x|^{\alpha} d y\right) \odot u, \tilde{o}\right) \\
& =\frac{1}{b-a} D\left(\left(\frac{(x-a)^{\alpha+1}+(b-x)^{\alpha+1}}{\alpha+1}\right) \odot u, \tilde{o}\right) \\
& =\frac{L_{f^{*}}}{b-a}\left(\frac{(x-a)^{\alpha+1}+(b-x)^{\alpha+1}}{\alpha+1}\right) .
\end{aligned}
$$

Next comes the basic Ostrowski type fuzzy result in

Theorem 5. let $f \in C^{1}\left([a, b], \mathbb{R}_{\mathcal{F}}\right)$, the space of one time continuously differentiable functions in the fuzzy sense. Then for $x \in[a, b]$,

$$
\begin{aligned}
& D\left(\frac{1}{b-a} \odot(F R) \int_{a}^{b} f(y) d y, f(x)\right) \\
& \quad \leq\left(\sup _{t \in[a, b]} D\left(f^{\prime}(t), \tilde{o}\right)\right)\left(\frac{(x-a)^{2}+(b-x)^{2}}{2(b-a)}\right) .
\end{aligned}
$$

Inequality (2) is sharp at $x=a$, in fact attained by $f^{*}(y):=(y-a)(b-a) \odot u$, $u \in \mathbb{R}_{\mathcal{F}}$ being fixed.

Proof. We observe that

$$
\begin{aligned}
D & \left(\frac{1}{b-a} \odot(F R) \int_{a}^{b} f(y) d y, f(x)\right) \\
& =D\left(\frac{1}{b-a} \odot(F R) \int_{a}^{b} f(y) d y, \frac{1}{b-a} \odot(F R) \int_{a}^{b} f(x) d y\right)
\end{aligned}
$$




$$
\begin{gathered}
=\frac{1}{b-a} D\left((F R) \int_{a}^{b} f(y) d y,(F R) \int_{a}^{b} f(x) d y\right) \\
\quad \underset{\leq}{(\text { by Lemma 2) }} \frac{1}{b-a} \int_{a}^{b} D(f(y), f(x)) d y
\end{gathered}
$$

$$
\begin{aligned}
& \underset{\leq}{(\text { by Theorem 2) }} \frac{1}{b-a} \int_{a}^{b}|y-x|\left(\sup _{t \in[a, b]} D\left(f^{\prime}(t), \tilde{o}\right)\right) d y \\
& =\frac{\left(\sup _{t \in[a, b]} D\left(f^{\prime}(t), \tilde{o}\right)\right)}{b-a}\left(\frac{(x-a)^{2}+(b-x)^{2}}{2}\right),
\end{aligned}
$$

proving (2).

By Propositions 1, 2 and Theorem 3 we get that $f^{* \prime}(y)=(b-a) \odot u$. We have that

$$
\begin{aligned}
\text { L.H.S.(2) } & =D\left(\frac{1}{b-a} \odot(F R) \int_{a}^{b}((y-a)(b-a) \odot u) d y, \tilde{o}\right) \\
& =D\left((F R) \int_{a}^{b}((y-a) \odot u) d y, \tilde{o}\right) \\
& =D\left(\left(\int_{a}^{b}(y-a) d y\right) \odot u, \tilde{o}\right) \\
& =D\left(\frac{(b-a)^{2}}{2} \odot u, \tilde{o}\right)=\frac{(b-a)^{2}}{2} D(u, \tilde{o}) .
\end{aligned}
$$

And

$$
\text { R.H.S. }(2)=\sup _{t \in[a, b]} D((b-a) \odot u, \tilde{o}) \frac{(b-a)}{2}=\frac{(b-a)^{2}}{2} D(u, \tilde{o}) .
$$

That is equality in (2) is attained.

We conclude with the following Ostrowski type inequality fuzzy generalization in

Theorem 6. Let $f \in C^{n+1}\left([a, b], \mathbb{R}_{\mathcal{F}}\right), n \in \mathbb{N}$, the space of $(n+1)$ times continuously differentiable functions on $[a, b]$ in the fuzzy sense. Call

$$
M:=\sum_{i=1}^{n} \frac{(b-a)^{i}}{(i+1) !} D\left(f^{(i)}(a), \tilde{o}\right) .
$$


Then

$$
\begin{aligned}
D & \left(\frac{1}{b-a} \odot(F R) \int_{a}^{b} f(x) d x, f(a)\right) \\
& \leq\left[M+\left(\sup _{t \in[a, b]} D\left(f^{(n+1)}(t), \tilde{o}\right)\right) \frac{(b-a)^{n+1}}{(n+2) !}\right] .
\end{aligned}
$$

If $f^{(i)}(a)=\tilde{o}, i=1, \ldots, n$. Then

$$
\begin{aligned}
& D\left(\frac{1}{b-a} \odot(F R) \int_{a}^{b} f(x) d x, f(a)\right) \\
& \quad \leq\left(\sup _{t \in[a, b]} D\left(f^{(n+1)}(t), \tilde{o}\right)\right) \frac{(b-a)^{n+1}}{(n+2) !} .
\end{aligned}
$$

Inequalities (3) and (4) are sharp, in fact attained by

$$
f^{*}(x):=(b-a)(x-a)^{n+1} \odot u, \quad u \in \mathbb{R}_{\mathcal{F}} \text { being fixed. }
$$

Corollary 2. Let $f \in C^{2}\left([a, b], \mathbb{R}_{\mathcal{F}}\right)$. Then

$$
\begin{aligned}
& D\left(\frac{1}{b-a} \odot(F R) \int_{a}^{b} f(x) d x, f(a)\right) \\
& \quad \leq\left[\frac{(b-a)}{2} D\left(f^{\prime}(a), \tilde{o}\right)+\left(\sup _{t \in[a, b]} D\left(f^{\prime \prime}(t), \tilde{o}\right)\right) \frac{(b-a)^{2}}{6}\right] .
\end{aligned}
$$

When $f^{\prime}(a)=\tilde{o}$, then

$$
\begin{gathered}
D\left(\frac{1}{b-a} \odot(F R) \int_{a}^{b} f(x) d x, f(a)\right) \\
\quad \leq\left(\sup _{t \in[a, b]} D\left(f^{\prime \prime}(t), \tilde{o}\right)\right) \frac{(b-a)^{2}}{6} .
\end{gathered}
$$

Proof of Theorem 6. Let $x \in[a, b]$, then by Theorem 1 we get

$$
f(x)=\sum_{i=1}^{n-1} f^{(i)}(a) \odot \frac{(x-a)^{i}}{i !} \oplus \mathcal{R}_{n}(a, x),
$$


where

$$
\left.\mathcal{R}_{n}(a, x):=(F R) \int_{a}^{x}\left(\int_{a}^{x_{1}} \cdots\left(\int_{a}^{x_{n-1}} f^{(n)}\left(x_{n}\right) d x_{n}\right) d x_{n-1}\right) \cdots\right) d x_{1}
$$

(here we need $x \geq a$ ). We observe that

$$
\begin{aligned}
& D\left(\frac{1}{b-a} \odot(F R) \int_{a}^{b} f(x) d x, f(a)\right) \\
& =\frac{1}{b-a} D\left((F R) \int_{a}^{b} f(x) d x,(F R) \int_{a}^{b} f(a) d x\right) \\
& =\frac{1}{b-a} D\left((F R) \int_{a}^{b}\left(\sum_{i=0}^{n-1} f^{(i)}(a) \odot \frac{(x-a)^{i}}{i !} \oplus \mathcal{R}_{n}(a, x)\right) d x,\right. \\
& \left.(F R) \int_{a}^{b} f(a) d x\right) \\
& =\frac{1}{b-a} \cdot D\left((F R) \int_{a}^{b}\left(\sum_{i=1}^{n-1} f^{(i)}(a) \odot \frac{(x-a)^{i}}{i !} \oplus \mathcal{R}_{n}(a, x)\right) d x, \tilde{o}\right) \\
& =\frac{1}{b-a} D\left((F R) \int_{a}^{b}\left(\sum_{i=1}^{n} f^{(i)}(a) \odot \frac{(x-a)^{i}}{i !} \oplus \mathcal{R}_{n}(a, x)\right) d x,\right. \\
& \left.(F R) \int_{a}^{b} f^{(n)}(a) \odot \frac{(x-a)^{n}}{n !} d x\right) \\
& =\frac{1}{b-a} D\left(\sum_{i=1}^{n}(F R) \int_{a}^{b} f^{(i)}(a) \odot \frac{(x-a)^{i}}{i !} d x\right. \\
& \left.\oplus(F R) \int_{a}^{b} \mathcal{R}_{n}(a, x) d x,(F R) \int_{a}^{b} f^{(n)}(a) \odot \frac{(x-a)^{n}}{n !} d x\right) \\
& =\frac{1}{b-a} D\left(\sum_{i=1}^{n} f^{(i)}(a) \odot \frac{(b-a)^{i+1}}{(i+1) !} \oplus(F R) \int_{a}^{b} \mathcal{R}_{n}(a, x) d x,\right. \\
& \left.(F R) \int_{a}^{b} f^{(n)}(a) \odot \frac{(x-a)^{n}}{n !} d x\right) \\
& \leq \frac{1}{b-a}\left[\sum_{i=1}^{n} \frac{(b-a)^{i+1}}{(i+1) !} D\left(f^{(i)}(a), \tilde{o}\right)\right. \\
& \left.+D\left((F R) \int_{a}^{b} \mathcal{R}_{n}(a, x) d x,(F R) \int_{a}^{b} f^{(n)}(a) \odot \frac{(x-a)^{n}}{n !} d x\right)\right]
\end{aligned}
$$




$$
\begin{aligned}
=M+ & \frac{1}{b-a} D\left((F R) \int_{a}^{b} \mathcal{R}_{n}(a, x) d x,(F R) \int_{a}^{b} f^{(n)}(a) \odot \frac{(x-a)^{n}}{n !} d x\right) \\
=M+ & \frac{1}{b-a} D\left(( F R ) \int _ { a } ^ { b } \left(\int _ { a } ^ { x } \left(\int_{a}^{x_{1}} \cdots\right.\right.\right. \\
& \left.\left.\left.\ldots\left(\int_{a}^{x_{n-1}} f^{(n)}\left(x_{n}\right) d x_{n}\right) d x_{n-1}\right) \cdots\right) d x_{1}\right) d x, \\
& \left.\left.\left.(F R) \int_{a}^{b}\left(\int_{a}^{x}\left(\int_{a}^{x_{1}} \cdots\left(\int_{a}^{x_{n-1}} f^{(n)}(a) d x_{n}\right) d x_{n-1}\right) \cdots\right) d x_{1}\right) d x\right)\right]
\end{aligned}
$$

(by Lemmas 2, 3)

$$
M+\frac{1}{b-a}\left[\int _ { a } ^ { b } \left(\int _ { a } ^ { x } \left(\int _ { a } ^ { x _ { 1 } } \cdots \left(\int _ { a } ^ { x _ { n - 1 } } D \left(f^{(n)}\left(x_{n}\right),\right.\right.\right.\right.\right.
$$

$$
\left.\left.\left.\left.\left.\left.f^{(n)}(a)\right) d x_{n}\right) d x_{n-1}\right) \cdots\right) d x_{1}\right) d x\right]
$$

$$
\begin{aligned}
& \underset{\leq}{\text { (by Theorem 2) }} M+\frac{1}{b-a}\left[\int _ { a } ^ { b } \left(\int _ { a } ^ { x } \left(\int _ { a } ^ { x _ { 1 } } \cdots \left(\int_{a}^{x_{n-1}}\left(x_{n}-a\right)\right.\right.\right.\right. \\
& \left.\left.\left.\left.\left.\cdot\left(\sup _{t \in[a, b]} D\left(f^{(n+1)}(t), \tilde{o}\right)\right) d x_{n}\right) d x_{n-1}\right) \cdots\right) d x_{1}\right) d x\right] \\
& =M+\frac{\left(\sup _{t \in[a, b]} D\left(f^{(n+1)}(t), \tilde{o}\right)\right)}{b-a} \frac{(b-a)^{n+2}}{(n+2) !} \\
& =M+\left(\sup _{t \in[a, b]} D\left(f^{(n+1)}(t), \tilde{o}\right)\right) \frac{(b-a)^{n+1}}{(n+2) !} .
\end{aligned}
$$

We have established inequalities (3) and (4).

Consider $g(x):=c(x-a)^{\ell} \odot u, x \in[a, b], c>0, \ell \in \mathbb{Z}_{+}, u \in \mathbb{R}_{\mathcal{F}}$ fixed. We prove that $g$ is fuzzy continuous. Let $x_{n} \in[a, b]$ such that $x_{n} \rightarrow x$ as $n \rightarrow+\infty$. Then

$$
\begin{aligned}
D\left(g\left(x_{n}\right), g(x)\right) & =D\left(c\left(x_{n}-a\right)^{\ell} \odot u, c(x-a)^{\ell} \odot u\right) \\
& \leq c\left|\left(x_{n}-a\right)^{\ell}-(x-a)^{\ell}\right| D(u, \tilde{o}) \rightarrow 0 .
\end{aligned}
$$

Hence by the last argument, Propositions 1, 2 and Theorem 3 we obtain that $f^{*} \in C^{n+1}\left([a, b], \mathbb{R}_{\mathcal{F}}\right)$.

We see that

$$
f^{*(i)}(a)=\tilde{o}, \text { for } i=1, \ldots, n
$$


That is $M=0$. Furthermore it holds

$$
f^{*(n+1)}(x)=(b-a)(n+1) ! \odot u .
$$

Finally, we notice that

$$
\begin{aligned}
\text { L.H.S. ((3), (4)) } & =D\left(\frac{1}{b-a} \odot(F R) \int_{a}^{b}\left((b-a)(x-a)^{n+1} \odot u\right) d x, \tilde{o}\right) \\
& =D\left(u \odot \int_{a}^{b}(x-a)^{n+1} d x, \tilde{o}\right)=D\left(u \odot \frac{(b-a)^{n+2}}{n+2}, \tilde{o}\right) \\
& =\frac{(b-a)^{n+2}}{n+2} D(u, \tilde{o}) .
\end{aligned}
$$

Also we find

R.H.S. ((3), (4)) $=(b-a)(n+1) ! D(u, \tilde{o}) \frac{(b-a)^{n+1}}{(n+2) !}=\frac{(b-a)^{n+2}}{n+2} D(u, \tilde{o})$.

Proving (3) and (4) sharp, in fact attained inequalities.

\section{REFERENCES}

[1] George A. Anastassiou, Ostrowski type inequalities, Proc. AMS, 123 (1995), 3775-3791.

[2] George A. Anastassiou, Rate of convergence of Fuzzy neural network operators, univariate case, accepted in Journal of Fuzzy Mathematics.

[3] George A. Anastassiou, Univariate fuzzy-random neural network approximation operators, submitted.

[4] George A. Anastassiou, On H-fuzzy differentiation, accepted in Mathematica Balkanica, (2002).

[5] George A. Anastassiou and Sorin Gal, On a fuzzy trigonometric approximation theorem of Weierstrass-type, in Journal of Fuzzy Mathematics, 9, No. 3 (2001), 701-708.

[6] A.M. Fink, Bounds on the deviation of a function from its averages, Czechoslavak Math. J., 42 (117) (1992), 289-310.

[7] S. Gal, Approximation theory in fuzzy setting, Chapter 13 in Handbook of Analytic Computational Methods in Applied Mathematics (edited by G. Anastassiou), Chapman \& Hall, CRC Press, Boca Raton, New York, 2000, pp. 617-666.

[8] A. Ostrowski, Über die Absolutabweichung einer differentiebaren Funktion von ihrem Integralmittelwert, Comment. Math. Helv., 10 (1938), 226-227. 
[9] M.L. Puri and D.A. Ralescu, Differentials of fuzzy functions, J. of Math. Analysis and Appl., 91 (1983), 552-558.

[10] Congxin Wu and Zengtai Gong, On Henstock integral of fuzzy number valued functions (I), Fuzzy Sets and Systems, 120, No. 3 (2001), 523-532.

[11] L.A. Zadeh, Fuzzy sets, Information and Control, 8 (1965), 338-353. 